\title{
A dynamical systems approach to the surface search for debris associated with the disappearance of flight MH370
}

\author{
V. J. García-Garrido ${ }^{1}$, A. M. Mancho ${ }^{1}$, S. Wiggins ${ }^{2}$, and C. Mendoza ${ }^{3}$ \\ ${ }^{1}$ Instituto de Ciencias Matemáticas, CSIC-UAM-UC3M-UCM. C/Nicolás Cabrera 15, Campus de Cantoblanco UAM, \\ 28049 Madrid, Spain \\ ${ }^{2}$ School of Mathematics, University of Bristol, Bristol, BS8 1TW, UK \\ ${ }^{3}$ ETSI Navales, Universidad Politécnica de Madrid, Av. Arco de la Victoria 4, 28040 Madrid, Spain
}

Correspondence to: A. M. Mancho (a.m.mancho@icmat.es)

Received: 7 July 2015 - Published in Nonlin. Processes Geophys. Discuss.: 27 July 2015

Revised: 30 October 2015 - Accepted: 13 November 2015 - Published: 25 November 2015

\begin{abstract}
The disappearance of Malaysia Airlines flight MH370 on the morning of 8 March 2014 is one of the great mysteries of our time. Perhaps the most relevant aspect of this mystery is that not a single piece of debris from the aircraft was found during the intensive surface search carried out for roughly 2 months following the crash. Difficulties in the search efforts, due to the uncertainty of the plane's final impact point and the time that had passed since the accident, bring the question on how the debris scattered in an always moving ocean, for which there are multiple data sets that do not uniquely determine its state. Our approach to this problem is based on the use of Lagrangian descriptors (LD), a novel mathematical tool coming from dynamical systems theory that identifies dynamic barriers and coherent structures governing transport. By combining publicly available information supplied by different ocean data sources with these mathematical techniques, we are able to assess the spatio-temporal state of the ocean in the priority search area at the time of impact and the following weeks. Using this information we propose a revised search strategy by showing why one might not have expected to find debris in some large search areas targeted by the Australian Maritime Safety Authority (AMSA), and determining regions where one might have expected impact debris to be located, which were not subjected to any exploration.
\end{abstract}

\section{Introduction}

The fate of Malaysia Airlines flight MH370 has been a mystery since its disappearance on the morning of 8 March 2014. An analysis of radar data, aircraft performance calculations and satellite communication (SATCOM) system signaling messages placed the aircraft over an arc, the seventh arc, in the southern part of the Indian Ocean (ATSB, 2014a), where the aircraft's fuel was, presumably, exhausted. Refinements to the analysis of both the flight and satellite data have been continuous since the disappearance of flight MH370 and have resulted in the definition of several potential impact regions along the seventh arc, driving the search efforts to consider different search areas.

The events determining the plane's fate after the crash were undoubtedly affected by the manner in which the impact occurred, since this should have determined the nature of the breakup of the plane into pieces of debris. A study by Chen et al. (2015) explored how different impact angles would have affected the breakup and also conjectured plane impact conditions for which no debris would have been produced. However, the discovery of a right wing flaperon from the plane on a beach of Réunion Island in the Indian Ocean, 16 months after the disappearance, has ruled out this possibility (http://edition.cnn.com/2015/09/03/ europe/mh370-investigation/index.html). On the other hand, search services always considered that, after the plane disappeared, floating debris should have been produced, and began one of the most challenging searches in aviation history. During roughly 2 months after the disappearance, a surface 
search was conducted. It was not until October 2014 that the much more demanding underwater search phase started.

It is hard to know what the properties of the debris produced after the impact should be, and some aspects in this respect are discussed by Chen et al. (2015). Large heavy pieces of the plane would have sank immediately, but small floating pieces are also expected. For instance, fuselage pieces similar to those recovered from the Air France Flight 447 flight, (see http://www.aviationlawmonitor.com/ tags/air-france-flight-447/), which consisted of thin, flat halfsubmerged structures, or personal flotation devices, which potentially would have been driven mainly by ocean currents. The presence of this kind of debris is consistent with the plan and actions taken by the search services and is confirmed by the discovery of a wing piece on the beach of Réunion Island.

Under these assumptions it is then clearly very important to understand the nature of the ocean currents in the impact area (Saab, 2014) at the time of disappearance and thereafter, and to then determine how they contributed to debris dispersion. We will assume that it is reasonable to relate the evolution of the described potential debris with that of surface drifters, such as those distributed by the Global Drifter Program (GDP), and for this reason, in order to support our study, we will benchmark our conclusions with these drifter tracks. It is important to remark that drifters are the only floating objects in the area, at the time of the impact, for which we have full knowledge of their time evolution. No comparison is possible with the real debris.

Differences between the forecasted velocity fields are a handicap for the analysis of particle dispersion, and therefore having a good representation of the ocean circulation patterns becomes crucial for dispersion studies (Mendoza et al., 2014; Griffa et al., 2013; Haza et al., 2007). Mainly two types of data sources for oceanic currents are available in oceanic contexts. One is obtained in almost real time after postprocessing satellite altimetry, such as Archiving, Validation and Interpretation of Satellite Oceanographic data (AVISO) products, which provide mainly geostrophic currents. Similar products integrating surface winds and/or Ekman dynamics are, for instance, Ocean Surface Current Analyses - Real Time (OSCAR) and Surface Current from Diagnostic model (SCUD).

Alternatively, surface ocean currents can be provided by physics-based computational models, which are solved on high-resolution meshes. These provide ocean circulation currents in space and time on the whole oceanic basin under study and, in order to be representative of the ocean state, they usually assimilate AVISO data and drifters and incorporate other important dynamics and measurements. Examples of these models are the Hybrid Coordinate Ocean Model (HYCOM), or the Operational Mercator global Ocean Analysis and Forecast System (provided by EU Copernicus). Typically these products provide high-resolution space-time data sets but only in areas of interest to the countries funding the consortia providing simulations. In this study the focus is in the middle of the Indian Ocean, where only low-resolution data are available for the considered models.

This study is focused in the analysis of AVISO and HYCOM data predictions for the region and period of interest. Our analysis utilizes fundamental ideas coming from dynamical systems theory applied to Lagrangian transport, which provide sharper insights than the simple drifter tracking supplied by tools such as General NOAA Operational Modeling Environment (GNOME) or SCUD. The approach taken by the rescue services as described in ATSB (2014a) seems to be closer to a simple drifter tracking approach, and no information is provided on the type of velocity fields used.

Dynamical systems theory contributes to this problem by realizing Poincaré's idea of seeking geometrical structures in the phase portrait (for this problem, the ocean surface) that can be used to schematically organize regions corresponding to qualitatively different types of trajectories. These geometrical objects, the stable and unstable manifolds of hyperbolic trajectories, also called Lagrangian structures, are expected to be robust with respect to slight perturbations of the velocity field. The main contribution from this perspective is the consideration that the analysis of individual trajectories may be misleading, and that the right approach to take is to observe the consistency of individual trajectories with the dynamical barriers highlighted by the Lagrangian skeleton of the velocity field.

Finding these structures in the context of geophysical flows is a dynamical systems challenge, as these velocity fields are time dependent and only known on a finite time data set. Their study requires the use of modern techniques in nonlinear dynamical systems theory that achieve a phase portrait representation for systems with these characteristics. In this context many tools have been successful to this end and have provided interesting insights into oceanic problems. For instance, manifolds have been approximated by computing ridges of fields, such as finite size Lyapunov exponents (FSLE) (Aurell et al., 1997) successfully applied into oceanic contexts (d'Ovidio et al., 2004; Tew Kai et al., 2009) and finite time Lyapunov exponents (FTLE) (Nese, 1989; Shadden et al., 2005, 2009). Another perspective within the geometrical approach different from Lyapunov exponents is that provided by distinguished hyperbolic trajectories (DHT), a generalization of the concept of fixed point for dynamical systems with a general time dependence (Ide et al., 2002; Ju et al., 2003; Madrid and Mancho, 2009), and their stable and unstable manifolds (Mancho et al., 2006c; Mendoza and Mancho, 2012). In this approach stable and unstable manifolds are directly computed as material surfaces (Mancho et al., 2003, 2004). This method has also provided valuable insight into oceanic problems (Mancho et al., 2006a; Mendoza et al., 2010). Other approaches in this field have been the geodesic theory of Lagrangian coherent structures (LCS) (Haller and Beron-Vera, 2012), the variational theory of LCS (Farazmand and Haller, 2012), the trajectory complexity measures (Rypina et al., 2011), mesohy- 
perbolicity measures and ergodic partitions (Mezic and Wiggins, 1999; Mezic et al., 2010) and transfer operator methods (Froyland and Padberg-Gehle, 2014; Froyland et al., 2012).

A recent tool that reveals phase space structures of general time dependent dynamical systems is the so-called function $M$, also referred to as Lagrangian descriptors (see for instance Mendoza and Mancho, 2010, 2012; Mancho et al., 2013), for which several advantages have been discussed in Mancho et al. (2013), Rempel et al. (2013) and de la Cámara et al. $(2012,2013)$ versus other classical approaches (Shadden et al., 2005; Mezic and Wiggins, 1999). We illustrate the power of this proposed methodology jointly with techniques based on contour advection (Dritschel, 1989; Mancho et al., 2003) to reach conclusions regarding the fate of the plane debris in the weeks after the accident.

In this paper we analyze the transport processes arising from AVISO and HYCOM velocity fields. In order to assess the performance (quality) of the ocean state data for the time period and region under consideration, we compute for each data set the Lagrangian geometrical skeleton by means of the function $M$, which is then benchmarked with surface GDP drifter tracks. We find that, remarkably, drifters follow the Lagrangian skeleton provided by AVISO data. HYCOM data present high-frequency motions, but our Lagrangian techniques still highlight mesoscale structures analogous to those found in AVISO. By combining contour advection of the presumed impact areas and the geometrical skeleton of the underlying flow, we note the presence of ocean mesoscale structures, which could have acted as barriers for the debris. Additionally, it is observed that the impact area evolves in such a way that it fills quiet ocean regions, suggesting that they are a very likely destination for the debris. Interestingly, our Lagrangian analysis reaches conclusions that could have guided the search tasks: it highlights regions disregarded by the search efforts, where debris could have been found, and points out that debris could scarcely have visited certain regions subjected to intense search.

The structure of this article is as follows. Section 2 describes the data used in this study. Section 3 describes and discusses the approach to the debris dispersion. Section 4 provides the results and the discussion. Finally, Sect. 5 provides the Conclusions.

\section{Data}

The surface velocity fields used for this work have been obtained from satellite altimetry produced by Ssalto/Duacs and distributed by AVISO, with support from CNES (Centre National d'Etudes Spatiales), and are downloadable from http:// www.aviso.oceanobs.com/duacs/. In particular we have considered the product Updated (UPD), which is reconstructed from four satellites. The data are given on a spatial grid of $1080 \times 915$ points (longitude/latitude). The latitude ranges from $82^{\circ} \mathrm{S}$ to $81.9746^{\circ} \mathrm{N}$ using a Mercator projection, and the longitude ranges from 0 to $359.667^{\circ}$ using a uniform grid. The spatial precision is thus $1 / 3^{\circ}$ at the Equator and is provided with daily frequency. AVISO data essentially reproduce geostrophic currents. A priori, the convenience of using this type data for our study is supported by work discussed in Olascoaga et al. (2013) and Mendoza et al. (2014), and eventually is confirmed by the results reported in Sect. 4.2.

A second source used for the velocity fields is the Hybrid Coordinate Ocean Model (HYCOM) (Bleck, 2002; Chassignet et al., 2007), which provides velocity fields throughout the entire ocean depth. This model assimilates satellite sea-surface height and temperature data by means of the Navy Coupled Ocean Data Assimilation (NCODA) system and it is forced by surface winds and air-sea fluxes. These factors introduce a high-frequency variability on HYCOM data, producing velocity fields that, at least in the very upper layers, are far from geostrophy. In the next section we will discuss issues of finite resolution and wind effects in relation to the debris dispersion problem. In particular, we have considered the HYCOM GLBa0.08 data set (global experiments expt_91.0 and expt91.1), which can be downloaded from http://hycom.org/dataserver/glb-analysis/. These experiments use 32 layers along the vertical coordinate and are carried out over a global Mercator curvilinear grid for the ocean, where latitude ranges from $78^{\circ} \mathrm{S}$ to $47^{\circ} \mathrm{N}$ and the equatorial resolution is $1 / 12^{\circ}$, resulting in a grid point spacing of approximately $7 \mathrm{~km}$ on average. Regions north of $47^{\circ} \mathrm{N}$ are represented with a bipolar patch. The data are provided daily.

In the calculations performed we attempt to filter the highfrequency variability by using the HYCOM data layer at $50 \mathrm{~m}$ depth. A similar depth choice for this model is used, for instance, in Sulman et al. (2013) or for CUPOM (Colorado University Princeton Ocean Model) in Branicki and Kirwan Jr. (2010). In the latter work, the authors show how the resulting Lagrangian structure is close to 2-D surfaces that extend nearly vertically into the water column. The full 3-D Lagrangian structures can be obtained by a vertical extension of the evolving stable and unstable manifolds calculated in the 2-D plane, but this is valid only throughout a layer whose thickness depends on the ratio of the characteristic velocity within the 2-D slice to the characteristic vertical shear of the horizontal velocities (Branicki et al., 2011; Branicki and Kirwan Jr., 2010). We confirm that this is the case in our study.

Finally, in order to benchmark the Lagrangian structures calculated for the velocity field data sets described above, surface drifter tracks are obtained from data distributed by the GDP, from the National Oceanic and Atmospheric Administration (NOAA) and Atlantic Oceanographic and Meteorological Laboratory (AOML). These tracks can be downloaded from http://www.aoml.noaa.gov/phod/dac/index.php. Each drifter has an identification number with five digits, which allows for the identification of different drifters. The sampling of the drifter position is once every $6 \mathrm{~h}$. GDP drifters are designed to avoid direct wind forcing and to rep- 
resent motions under currents at a nominal depth of $15 \mathrm{~m}$ (Grodsky et al., 2011). This supports the comparison of their motion with AVISO and with HYCOM data in a layer below the surface as the one chosen.

\section{Modeling debris dispersion}

The debris produced after a plane accident is due to the breakup of the plane and it depends on how it enters the water. Large, heavy and unbroken pieces of fuselage would sink rapidly; however, reports exist of plane accidents (Chen et al., 2015) that have produced debris spread over a wide area, with light pieces that might have floated for a long time. The discovery of a right wing flaperon from the plane on a beach of Réunion Island the 29 July 2015 confirms this point.

This article is focused on discussing the search strategy, from the dynamical systems point of view, of floating debris that does not surpass the waterline and is not subjected to wind sailing effects. This debris would have been mainly driven by ocean currents, similarly to GDP drifters, i.e. we will assume a similarity between this debris and drifter motion. In our approach we disregard the direct action of the wind. However, HYCOM data incorporate this effect on the ocean surface, and thus our study does not neglect this effect completely.

It is clear that wind action is not negligible when considering the leeway of different kinds of objects. Works devoted to exploring this contribution are for instance Breivik and Allen (2008) and Breivik et al. (2011). As mentioned in these works, wind action is strongly dependent on the shape and size of the object and on how it surpasses the waterline. Thus, an accurate study requires previous knowledge of the objects themselves, which is not known in this case (except for the flaperon). In this respect, our study addresses the scattering of flat and thin objects below the waterline, which could have only been driven by ocean currents.

A perspective similar to the one in this article has been taken by Kuznetsov et al. (2002), who have examined the role played by Lagrangian structures obtained in the Gulf of Mexico. In particular, they have analyzed data from the Colorado University Princeton Ocean Model and have compared the geometry of the dynamics with simultaneous trajectories of drifters measured in an independent way. Indirectly, the agreement between the drifter evolution and the Lagrangian patterns of purely advected particles has served as a confirmation of the model quality. Also, Beron-Vera et al. (2010) compare the quality of two different altimetry AVISO products based on the agreement between a drifter trajectory and the Lagrangian skeleton supplied by the FTLE.

Recent studies (Tallapragada and Ross, 2008; Beron-Vera et al., 2015) have considered deviations in the evolution of drifters from that of purely advected particles by taking into account inertial effects produced by the buoyancy of the object and their finite size. Their approach considers the Maxey-Riley equation (Maxey and Riley, 1983), which holds for small rigid spheres. Similarly to wind effects, inertial effects depend on the shape of the debris objects, which we do not know in this case, except for the flaperon piece. Although beyond the scope of this work, a thorough study of inertial effects for the flaperon would be of much interest, as it would help to track back, with a larger degree of accuracy, a possible impact point. The lack of knowledge on the distribution of shapes, densities and sizes of the floating debris requires too many assumptions, which would prevent a reliable conclusion on the inertial effects. We thus simplify our approach to that of purely advected particles. In the end, the agreement found between the GDP drifter tracks and the mesoscale features that our tools highlight supports this decision. In this way we consider the motion of the airplane debris as that of purely advected particles. Such particles follow trajectories $\boldsymbol{x}(t)$ in the ocean that evolve according to the dynamical system:

$$
\frac{\mathrm{d} \boldsymbol{x}}{\mathrm{d} t}=\boldsymbol{v}(\boldsymbol{x}(t), t),
$$

where $\boldsymbol{v}(\boldsymbol{x}(t), t)$ is the velocity field of the ocean region of interest. In our analysis, as previously explained, we will assume that the motion of particles is mainly horizontal. The equations of motion that describe the horizontal evolution of particle trajectories on a sphere of radius $R$ are

$$
\begin{aligned}
\frac{\mathrm{d} \lambda}{\mathrm{d} t} & =\frac{u(\lambda, \phi, t)}{R \cos \phi}, \\
\frac{\mathrm{d} \phi}{\mathrm{d} t} & =\frac{v(\lambda, \phi, t)}{R} .
\end{aligned}
$$

Here the variables $(\lambda, \phi)$ are longitude and latitude, $u$ and $v$, respectively, represent the eastward and northward components of the velocity field provided by the data set.

For our Lagrangian analysis, which is based on Lagrangian descriptors (function $M$ ) and also on contour evolution, it is necessary to integrate Eqs. (2)-(3) in order to compute particle trajectories. As the velocity field for the system Eqs. (2)-(3) is provided solely on a discrete space-time grid, the first issue to deal with is that of interpolation. We have used bicubic interpolation in space and third-order Lagrange polynomials in time according to the details given in Mancho et al. (2006b) and Mendoza et al. (2014). This assumes smoothness of the velocity field below the resolution, which, on the other hand, is rather low.

An open question here is how robust are Lagrangian techniques when dealing with ocean motions that do not vary smoothly at small scales, as those having an inherent turbulent nature. Recent work by Hernández-Carrasco et al. (2011) analyzes this question by looking at the impact on FTLE of the two major effects when dealing with real data, namely, noise and dynamics of unresolved scales. Their work concludes that, although some features are lost, this tool still gives an accurate picture of the oceanic transport properties. 
We take these conclusions as supportive of our assumptions in the interpolation scheme that we use. On the other hand, effects of high-frequency variations on the velocity field are also included in the HYCOM data. In the last section we will highlight common features between this and the more smooth AVISO data, which will also support the conclusions in Hernández-Carrasco et al. (2011).

\section{Results and discussion}

\subsection{The Lagrangian skeleton}

The global nature of particle trajectories generated by Eq. (1) can be understood through the spatio-temporal template formed by geometrical structures of the flow that organize trajectories into distinct ocean regions, corresponding to qualitatively different types of trajectories. The boundaries or barriers between these regions are time-dependent material surfaces (which, mathematically, are invariant manifolds). Such a spatio-temporal template can be constructed with the technique that is referred to as Lagrangian descriptors (LD), based on a function, function $M$ (see Madrid and Mancho, 2009; Mendoza and Mancho, 2010; Mancho et al., 2013), defined as follows:

$M\left(\boldsymbol{x}_{0}, t_{0}, \tau\right)=\int_{t_{0}-\tau}^{t_{0}+\tau}\left\|\boldsymbol{v}\left(\boldsymbol{x}\left(t ; \boldsymbol{x}_{0}\right), t\right)\right\| \mathrm{d} t$,

where || || stands for the modulus of the velocity vector. At a given time $t_{0}$, function $M\left(\boldsymbol{x}_{0}, t_{0}\right)$ measures the arc length traced by the trajectory starting at $\boldsymbol{x}_{0}=\boldsymbol{x}\left(t_{0}\right)$ as it evolves forwards and backwards in time for a time period $\tau$. The structure of the function $M$ shows, at low $\tau$ values, a smooth pattern such as that visible in Fig. 1a. There, $M$ has been evaluated for AVISO data, on the date of the plane crash and near one of the search areas, using an integration period of $\tau=5$ days. On the other hand, Fig. 1b (computed for $\tau=20$ days) illustrates how the structure of $M$ evolves for large $\tau$ towards less regular structures. By this we mean that sharp changes of $M$ values occur in narrow gaps, forming filaments that highlight stable and unstable manifolds. A thorough explanation of this effect is discussed in Mendoza and Mancho (2010, 2012) Mancho et al. (2013) and Lopesino et al. (2015). Figure 1c shows a different projection for the latter case, which highlights the singular features of $M$ aligned with stable and unstable invariant manifolds, and their crossing in a hyperbolic point. We remark that the information contained in these figures is obtained by means of integration of particle trajectories. In fact some of the figures reported in this article use tens of thousands of them. The difference in this analysis with respect to that performed by the drifter tracking approach taken by ATSB (2014b), or other tools such as GNOME or SCUD, is the way in which this information is displayed. The latter tend to represent this output as spaghetti diagrams, while our Lagrangian perspective finds geometrical structures on the ocean surface, which are used to distinguish regions corresponding to qualitatively different types of trajectories.

Large $M$ values, represented in reddish colors, are related to regions of high-speed fluid (such as jets), while bluish colors denote calm regions. Figure 1d schematically shows the manifold skeleton and how particles, such as drifters or plane debris would evolve in the neighborhood of this structure. Invariant manifolds are dynamic barriers that cannot be crossed by purely advected particles and control transport in the region. The cross point of the stable and unstable manifolds, the hyperbolic point, drastically bends the trajectories. Tracer blobs evolve constrained by the manifold structure. Figure 1e illustrates this point by showing elongation of blob contours along the unstable manifold of a hyperbolic trajectory and stretching along its stable manifold. It is important to remark here that, from the dynamical system perspective, the main distortion agents for the contours are those causing their filamentous shape and, as explained here, these agents are the hyperbolic trajectories and their stable and unstable manifolds. Additionally, in the flow there exists regions dominated by trajectories with elliptic type stability, in which, contrary to what happens near hyperbolic trajectories, blobs remain coherent instead of forming filaments. This is illustrated in Fig. 1f. Oceanic eddies are an example of regions displaying such a behavior (see, e.g. Chelton et al., 2011) and this is also the case for oceanic jets, in which blobs are transported at a high speed but not distorted into filaments (see e.g. Wiggins and Mancho, 2014). Therefore, the interplay between stretching and confinement is an essential ingredient of fluid transport processes.

In this work, the importance of how water masses evolve is clear because of the uncertainty of the plane's impact point, and thus, in order to reach conclusions on the fate of the plane debris, it is important to track the time evolution of areas in which the plane potentially could have crashed, and recognize the constraints on these regions found in the ocean during the period after the accident. The evolution of these areas is tracked with the contour advection algorithm developed by Dritschel (1989), but including some modifications explained in Mancho et al. (2003), Mancho et al. (2004) and Mancho et al. (2006c).

\subsection{Analysis of the MH370 plane debris dispersion}

The goal of this section is to analyze the search strategy followed by the Australian Maritime Safety Authority (AMSA) in the months just after the crash, in order to see if, in light of the tools and data described in the previous sections, alternative search strategies could have been suggested.

One of the major challenges in the first stages of the search was to know how debris had scattered in an always moving ocean. On Tuesday 18 March and on Wednesday 19 March search areas were defined by AMSA according to possible 
(a)

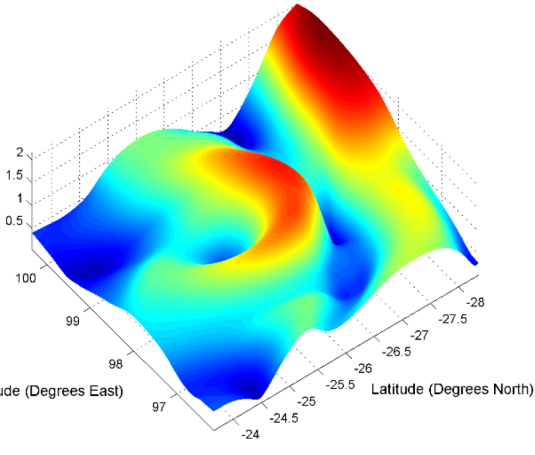

(c)

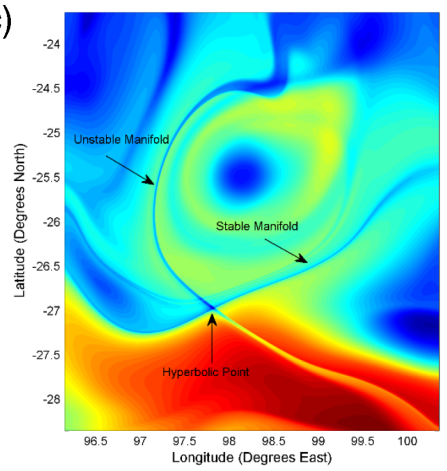

(b)

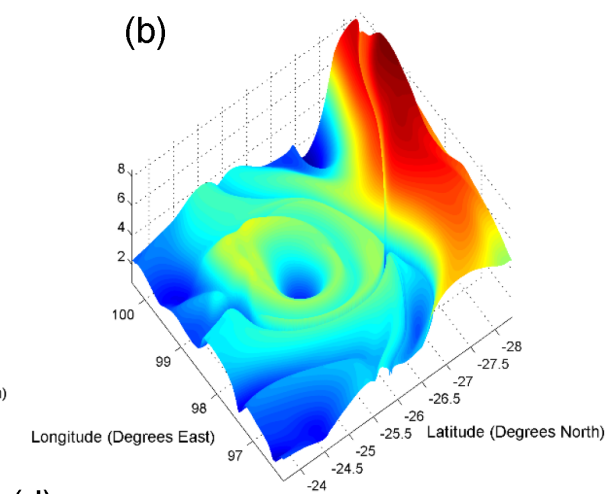

(d)

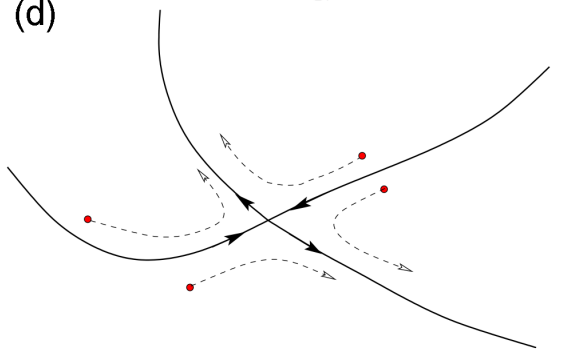

(f)

e)
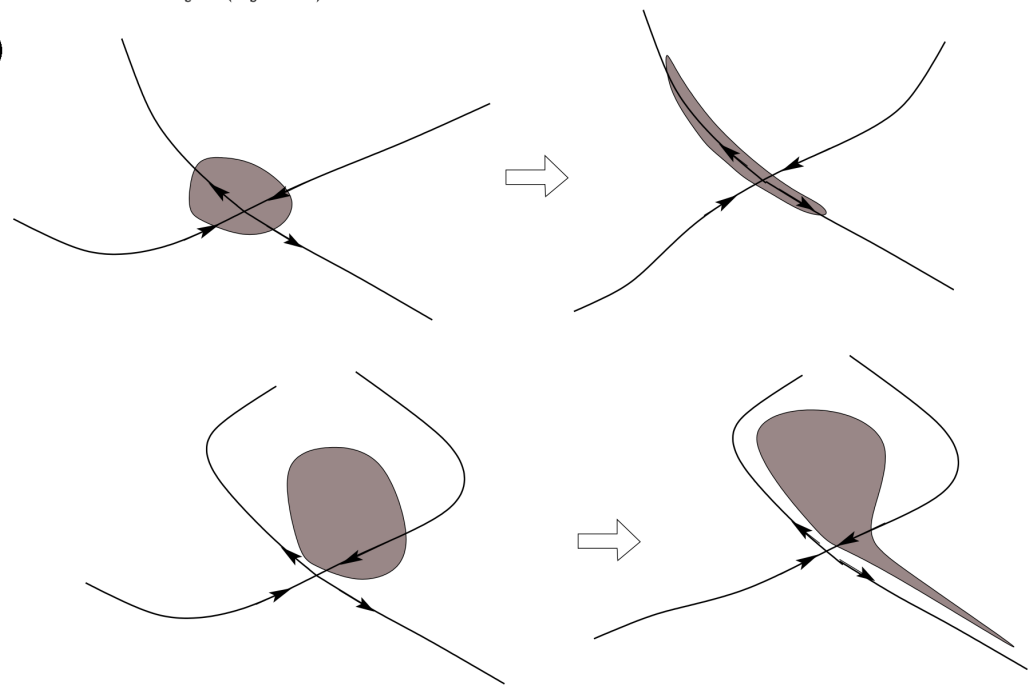

Figure 1. Evaluation of the $M$ function for AVISO data in the southern Indian Ocean on the 8 March 2014: (a) $\tau=5$ days, (b) $\tau=20$ days, (c) a countour plot of (b) highlighting the position of visible invariant manifolds and a hyperbolic point, (d) a schematic representation of the manifolds of (c) showing the particle evolution in the neighborhood, (e) tracer blob evolution in a neighborhood of the crossing of the stable and unstable manifolds and (f) tracer blob evolution in a neighborhood of a hyperbolic point and a elliptic (eddy-like) region.

flight paths deduced from satellite and aircraft performance data, which assumed impact points along the seventh arc. The charts released by AMSA for the different surface search areas are available at http://www.amsa.gov.au/media/incidents/ mh370-search.asp. Figure 2a shows the potential flight paths in white, and the seventh arc in black. The presumable impact area along the seventh arc is depicted in pink. Its width, which is at around $93 \mathrm{~km}$, expresses uncertainties in the impact point. Our discussion is based on this uncertainty band, which was considered as the maximum priority in the reports by ATSB (2014a, b). However, we note that the satellite analysis also has highlighted a wider search band around this narrower strip.

The search area released by AMSA during these days appears in the figure surrounded by a dashed line. Moreover, the gray shaded contour shown in this figure represents the advection of the presumed initial impact area until 19 March, according to ocean velocity data distributed by AVISO. This 

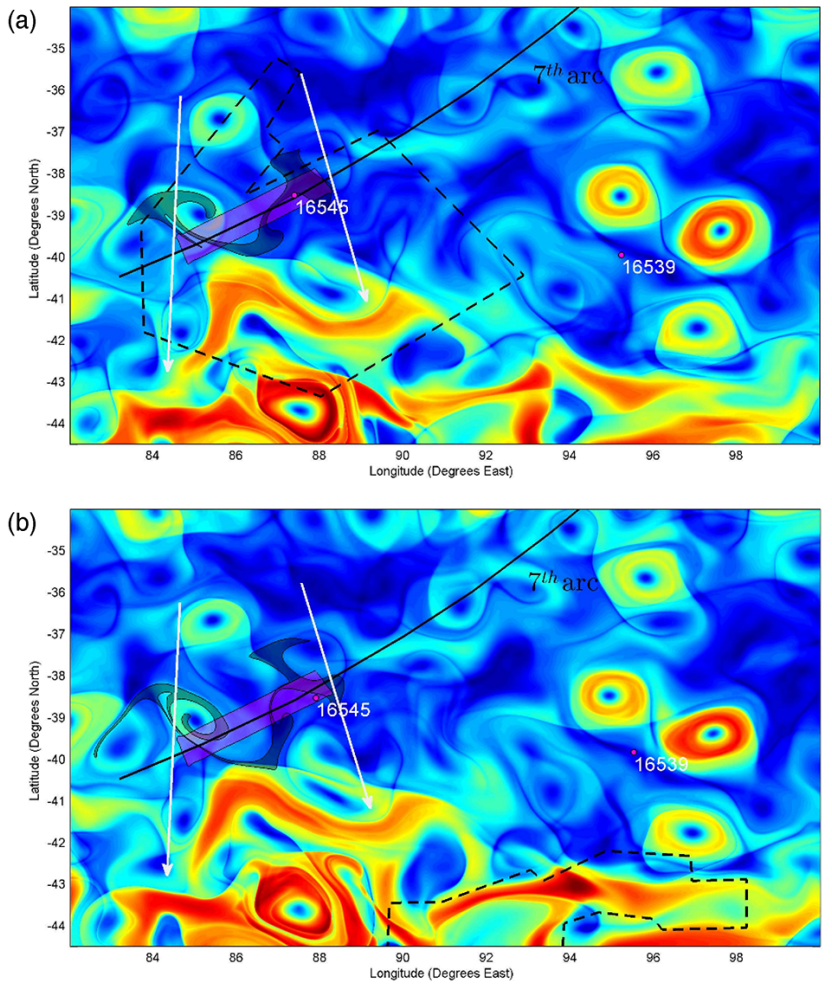

Figure 2. A summary of the debris search for the Malaysian MH370 plane in the southern Indian Ocean. In the background the $M$ function, evaluated on AVISO data for $\tau=20$ days, highlights mesoscale structures and invariant manifolds. Overlapped are the drifters in the area (magenta dots), the expected flight paths (white arrows), the likely impact area (pink) and its contour evolution (gray). Dashed lines outline the search areas for the respective dates, (a) 19 March 2014 and (b) 27 March 2014.

analysis thus suggests a smaller search area than the one determined by AMSA for the search operations that took place during 18 and 19 March. However, one remaining question that needs to be addressed in order to support this conclusion is, how accurate are AVISO data when representing the true ocean state? We provide some discussion of this point in terms of the tools described in the previous subsection. We observe that the advected contour stretches along unstable manifolds, which act as the main deforming agents for this blob. Stable and unstable manifolds and eddies are recognized in the background of Fig. 2a by means of the function $M$. The dark blue regions represent calm ocean regions and their presence is consistent with drifter motions. For instance, drifter 16545, represented by a magenta dot on 19 March, appears to be in one of these calm regions, and consistently it barely moves. Video $\mathrm{S} 1$ in the Supplement confirms that this is the case from 8 to 20 March. Similarly, the movie confirms that the drifter 16539 placed in a zone further east, during the period running from 2 to 27 March remains in a blue calm region, scarcely moving, consistent with AVISO data. The agreement between AVISO data and drifter motions supports
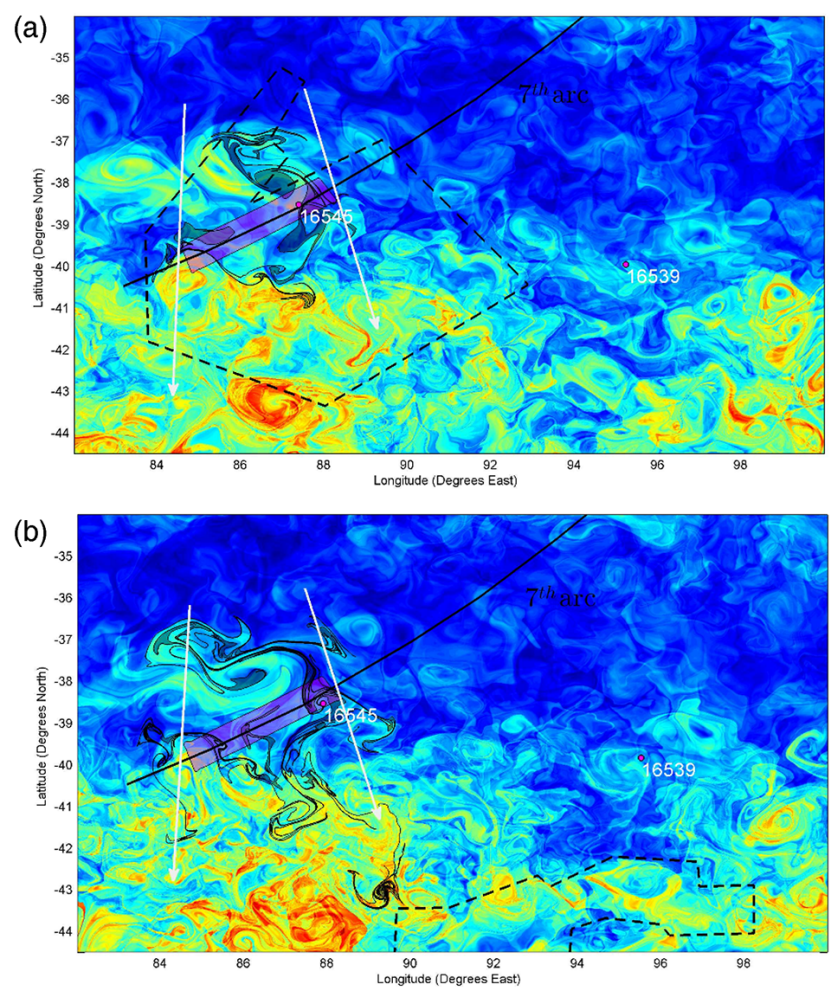

Figure 3. A summary of the debris search for the Malaysian MH370 plane in the southern Indian Ocean. In the background the $M$ function, evaluated on HYCOM data for $\tau=15$ days at $50 \mathrm{~m}$ depth, highlights mesoscale structures and invariant manifolds. Overlapped are the drifters in the area (magenta dots), the expected flight paths (white arrows), the likely impact area (pink) and its contour evolution (gray). Dashed lines outline the search areas for the respective dates, (a) 19 March 2014 and (b) 27 March 2014.

the adequacy of AVISO to accurately describe the ocean motion.

Satellite imagery provided to AMSA on 20 March revealed objects that could have been possible debris of the missing Malaysia Airlines flight MH370. Subsequently, the search area was shifted $185 \mathrm{~km}$ to the southeast of the original search area and the search efforts were focused in this area from 20 to 27 March. This new search area is shown in Fig. $2 b$ limited by a dashed line. Drifter 16539 is now the closest to the new search area. The gray shaded contour represents the advection of the presumed initial impact area along the seventh arc until 27 March 2014, and the coloring at the background highlights for this day, by means of the $M$ function, the Lagrangian skeleton. The figure suggests that according to AVISO the debris of a plane that crashed along the seventh arc could not have flowed to this region in this period of time, and thus the objects detected in this area should have had a different origin.

Figure $3 \mathrm{a}$ and $\mathrm{b}$ proceed with a similar analysis to that of Fig. 2a and b, but using HYCOM data instead. In the HYCOM simulations, layers near the surface are strongly dom- 
inated by wind forcing and, as a result, the velocity fields are subjected to high-frequency variations. As explained in Sects. 1 and 2, our study assumes a similarity between debris motion and that of drifters. Mancho et al. (2006a), Branicki and Kirwan Jr. (2010) and Sulman et al. (2013) justify the study of Lagrangian structures from velocity fields produced by models by considering layers just below the very upper layers that are slightly wind filtered. In Fig. 3a and b the background coloring show 2-D Lagrangian structures obtained at $50 \mathrm{~m}$ depth. The Lagrangian structures now are much more blurred than those obtained from AVISO, but still a comparison is feasible in terms of the mesoscale structures highlighted by the $M$ function. HYCOM shows mesoscale features linked to the circumpolar current further spread into the north, and the calm region close to drifter 16545 still seems to be present, but rather diffuse and displaced south of the drifter. Figure $3 \mathrm{a}$ illustrates that the spreading of the presumed impact area is broader than that computed from AVISO data, but still smaller than the AMSA search area. Further details are found in movie S2. The calm area in which drifter 16539 seemed to be anchored, according to Fig. 3b, now appears as a more active region with diffuse structures. However, the HYCOM analysis likewise confirms that the debris of a plane crashing along the seventh arc could have barely reached this region in this period of time.

On 28 March further refinements of the flight path shifted the search area up to the north, and the newly determined most likely impact area was then the one depicted in Fig. 4a (ATSB, 2014a) in the northeast direction above the Broken Ridge along the seventh arc. From 28 March to 3 April the search services were focused on the area surrounded by the dashed line in Fig. 4a. The background coloring of this figure shows the Lagrangian structures obtained from the $M$ function evaluated from AVISO data on 3 April 2014. The presumed initial impact area is advected until this day and represented in the gray shaded region. Its filamented structures clearly tend to be aligned along the unstable manifolds visible in the singular features of $M$. Numerous drifters are represented with magenta dots and they move consistently with the highlighted Lagrangian skeleton. This is further confirmed in video S3. For instance drifter 56566, which on the day of the accident, is within a strong mesoscale structure, an oceanic eddy placed at the east of the presumed impact area, and remains within the eddy during several months, being transported with it towards the west. Similarly, drifters 56947 and 56512 are trapped at some point in weaker eddies. Drifters 56557 and 56502, navigate along stable or unstable manifolds during noticeable periods. The latter drifter, which was placed very close to the presumed search area on the impact day, remains close to the contour after this time interval.

The strong eddy containing the drifter 56566 behaves like a capsule preventing the surrounding waters from penetrating. The advected gray shaded region deforms around it, showing it is a forbidden region for the debris. On 3 April, (a)
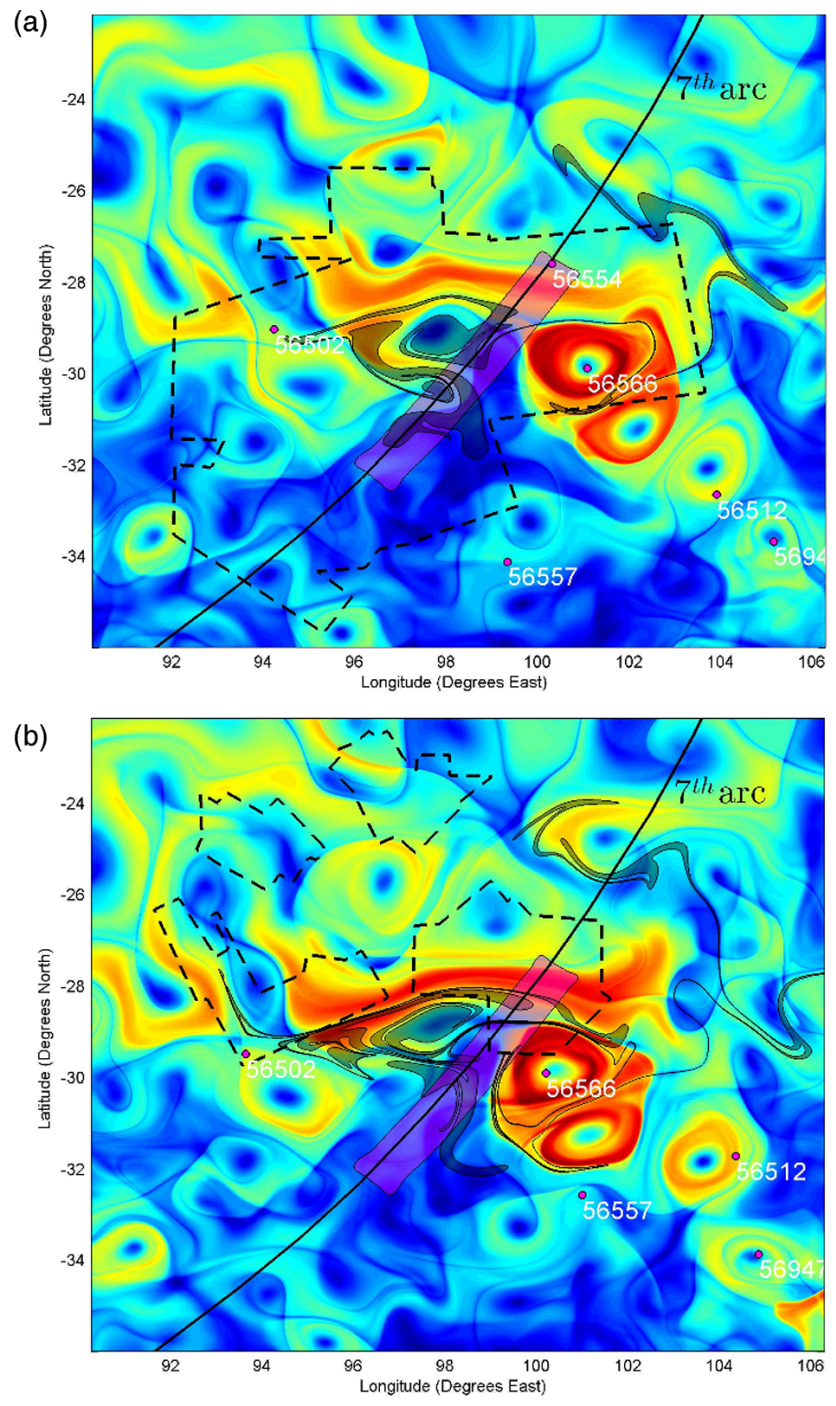

Figure 4. A summary of the debris search for the Malaysian MH370 plane at the north of the Broken Ridge in the Indian Ocean. In the background the $M$ function, evaluated on AVISO data for $\tau=20$ days, highlights mesoscale structures and invariant manifolds. Overlapped are the drifters in the area (magenta dots), the likely impact area (pink) and its contour evolution (gray). Dashed lines surround the search areas for the respective dates, (a) 3 April 2014 and (b) 15 April 2014.

this large vortex invaded the search area, and thus our analysis suggests that the region occupied by this moving coherent structure could have been removed from the region where the search efforts were focused.

Another interesting mesoscale element in this area is the strong jet, which crosses the presumed impact area at the north end. The feature is visible in orange-reddish colors and its presence is confirmed by the motion of drifter 56554, which navigates towards the east between 27 March and 11 April. The jet persists from the impact day (the 8 March) until at least the end of May and as we will discuss below is 
a dynamic barrier. It separates the blob located in the impact area into two pieces, which remain unmixed for the entire period of study, a small one at its north side and the larger one at south side.

Between 3 and 11 April the search area was moved up to the north, to regions rather diverted from the probable impact area displayed in Fig. 4a. We ignore this part and we pursue our discussion with the search targets held from 12 to 28 April, which are consistent with the impact area under consideration. The search areas after 12 April are reported by the Australian government (see at http://www.jacc.gov. $\mathrm{au} / \mathrm{media} / \mathrm{releases} / 2014 / \mathrm{april} / \mathrm{index}$.aspx), and correspond to the regions surrounded by dashed lines in Fig. 4b. There, it is shown again the presumed impact area along the seventh arc, and the advection of this contour by 15 April. From the background coloring of the $M$ function, we conclude that as time evolves the contour tends to form filaments aligned with the unstable manifolds and accumulates into calm regions, which gather most of the material (see also the video S3). From the figure it is clear that most of the impact area has scattered into the calm region below the strong jet described in the previous paragraph. Interestingly, this region was left out of the search area for this period, and reversely the search area barely contains material coming from the impact area. As time evolves the gray shaded contour evolves and penetrates more and more into the search panel at the right, but just at the south of the strong jet. We see that the jet acts as a barrier, which prevents most of the material of the likely impact area from being transported towards the north, this had the effect of paradoxically concentrating the main search in an area where, according to our analysis, there was likely no debris.

Figure $5 \mathrm{a}$ and $\mathrm{b}$ show a similar analysis to that of Fig. $4 \mathrm{a}$ and $\mathrm{b}$ using HYCOM data at $50 \mathrm{~m}$ depth. Mesoscale structures in this data are consistent with those of AVISO. For instance, the large eddy that isolates drifter 56566 is present in a similar position, invading the search area from 28 March to 3 April, confirming again that this part could have been excluded from the search. The jet structure splitting the impact region in two is also present at the same position, although somewhat perturbed by wind effects. Similar to AVISO, much of the material contained in the impact area would evolve filling calm regions that, roughly speaking, are positioned the same. The analysis of Fig. $5 \mathrm{~b}$ is similar to that of Fig. $4 \mathrm{~b}$, and it says that, according to our diagnosis, on the 15 April most of the material in the impact area is outside the search area. Video S4 completes these conclusions.

\section{Conclusions}

This work addresses, from the dynamical systems perspective, the fate of the MH370 plane debris after its presumed crash on the morning of 8 March 2014. This point of view provides a powerful tool for dealing with the difficulty aris-
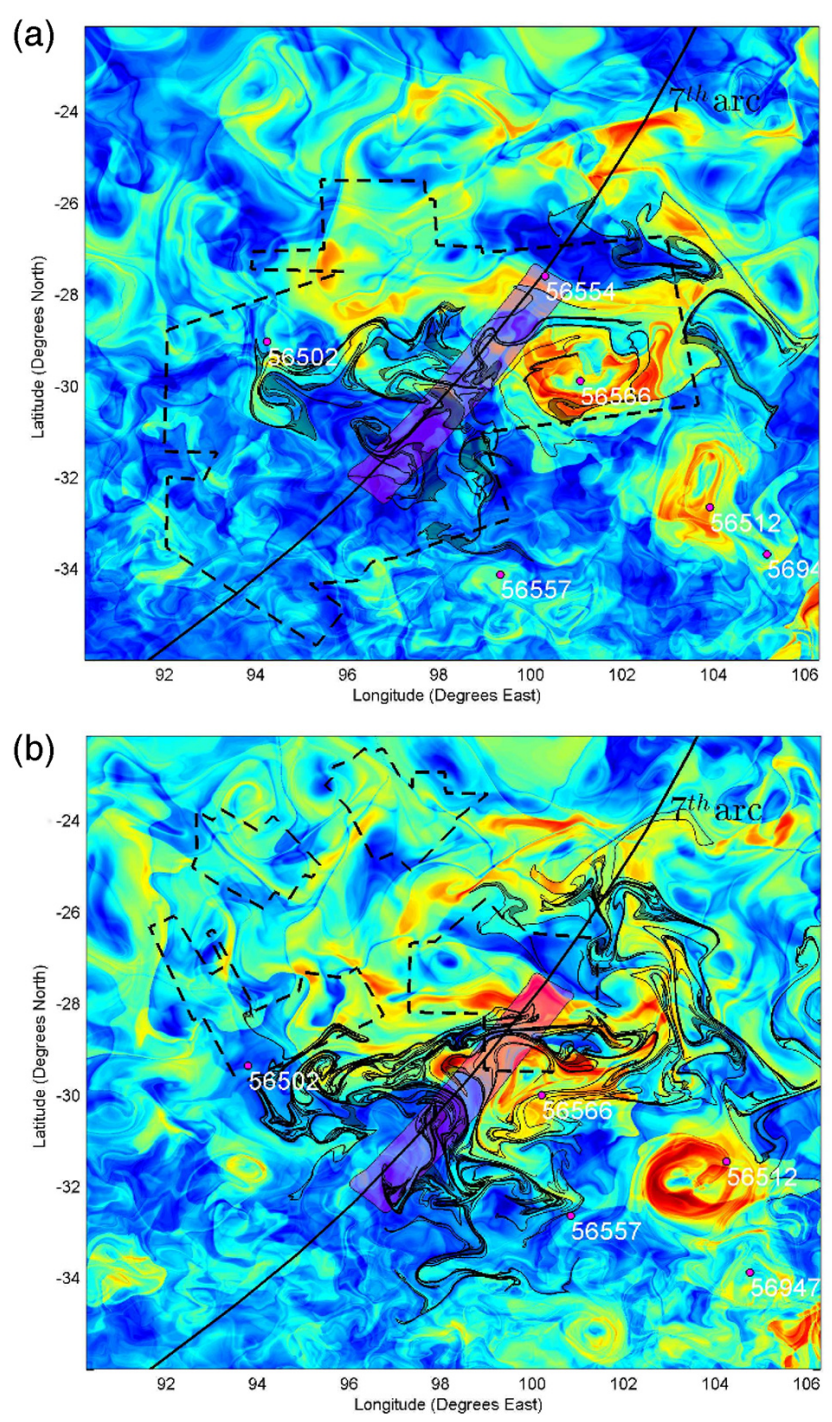

Figure 5. A summary of the debris search for the Malaysian MH370 plane at the north of the Broken Ridge in the Indian Ocean. In the background the $M$ function, evaluated on HYCOM data for $\tau=15$ days at $50 \mathrm{~m}$ depth, highlights mesoscale structures and invariant manifolds. Overlapped are the drifters in the area (magenta dots), the likely impact area (pink) and its contour evolution (gray). Dashed lines surround the search areas for the respective dates, (a) 3 April 2014 and (b) 15 April 2014.

ing from the uncertainty of predictions, which comes from the fact that there are several data sets that define multiple ocean states. Additional uncertainties coming from wind and inertial effects have not been taken into account, as too many assumptions on the shape, size and densities of the debris objects would have been required, preventing a reliable conclusion in this regard. Drifters are the only floating objects at the time of the impact for which we have full knowledge of their position and progress, and they evolve mainly trans- 
ported by the ocean currents. Our conclusions are backed by comparisons with their evolution.

The findings described in this paper highlight several facts that could have been relevant to the search for debris associated with flight MH370, subject to the restrictions just mentioned. First, we have shown that, according to several available data sources, impact debris from the MH370 plane would have been mainly scattered into calm ocean regions since the material from the likely impact areas tends to fill these areas. Second, mesoscale structures such as ocean eddies and jets may have played a key role in determining forbidden regions and barriers for debris. The consistency found between data obtained from different and independent sources (AVISO, HYCOM, drifters) back the evidence that the mesoscale structures we have described were present in the Indian Ocean during the accident time frame, and that considering these structures could have guided an efficient search procedure. These facts also back the reliability of our dispersion analysis, which would have suggested channeling efforts to regions disregarded from scheduled search areas and bypassing certain regions that were subjected to intense search.

\section{The Supplement related to this article is available online at doi:10.5194/npg-22-701-2015-supplement.}

Acknowledgements. V. J. García-Garrido, A. M. Mancho and C. Mendoza are supported by MINECO grant MTM2014-56392-R. The research of S. Wiggins is supported by ONR grant no. N0001401-1-0769. We acknowledge support from MINECO: ICMAT Severo Ochoa project SEV-2011-0087. Thanks are owed to CESGA and ICMAT for computing facilities.

Edited by: R. Grimshaw

Reviewed by: two anonymous referees

\section{References}

ATSB: Australian Transport Safety Bureau: MH370 Definition of Underwater Search Areas, available at: http://www.atsb.gov.au/media/5243942/ae-2014-054_mh370_ -_definition_of_underwater_search_areas_18aug2014.pdf (last access: 29 July 2015), 2014a.

ATSB: Australian Transport Safety Bureau: MH370 - Flight Path Analysis Update, available at: http://www.atsb.gov.au/media/ 5163181/AE-2014-054_MH370_-FlightPathAnalysisUpdate. pdf (last access: 29 July 2015), 2014b.

Aurell, E., Boffeta, G., Crisanti, A., Paladin, G., and Vulpiani, A.: Predictability in the large: An extension of the concept of Lyapunov exponent, J. Phys. A, 30, 1-26, 1997.

Beron-Vera, F. J., Olascoaga, M. J., and Goni, G. J.: Surface Ocean Mixing Inferred from Different Multisatellite Altimetry Measurements, J. Phys. Oceanogr., 40, 2466-2480, 2010.
Beron-Vera, F. J., Olascoaga, M. J., Haller, G., Farazmand, M., Trinanes, J., and Wang, Y.: Dissipative inertial transport patterns near coherent Lagrangian eddies in the ocean, Chaos, 25, 087412, doi:10.1063/1.4928693, 2015.

Bleck, R.: An oceanic general circulation model framed in hybrid isopycnic-Cartesian coordinates, Ocean Model., 4, 55-88, 2002.

Branicki, M. and Kirwan Jr., A. D.: Stirring: The Eckart paradigm revisited, Int. J. Eng. Sci., 48, 1027-1042, 2010.

Branicki, M., Mancho, A. M., and Wiggins, S.: A Lagrangian description of transport associated with a Front-Eddy interaction: application to data from the North-Western Mediterranean Sea, Physica D, 240, 282-304, 2011.

Breivik, O. and Allen, A. A.: An operational search and rescue model for the Norwegian Sea and the North Sea, J. Mar. Syst., 69, 99-113, 2008.

Breivik, O., Allen, A. A., Maisondieu, C., and Roth, J. C.: Windinduced drift of objects at sea: The leeway field method, Appl. Ocean Res., 33, 100-109, 2011.

Chassignet, E. P., Hurlburt, H. E., Smedstad, O. M., Halliwell, G. R., Hogan, P. J., Wallcraft, A. J., Baraille, R., and Bleck, R.: The HYCOM (HYbrid Coordinate Ocean Model) data assimilative system, J. Mar. Syst., 65, 60-83, doi:10.1016/j.jmarsys.2005.09.016, 2007.

Chelton, D. B., Schalx, M. G., and Samelson, R. M.: Global observations of nonlinear mesoscale eddies, Prog. Oceanogr., 91, 167-216, doi:10.1016/j.pocean.2011.01.002, 2011.

Chen, G., Gu, C., Morris, P. J., Paterson, E. G., Sergeev, A., Wang, Y.-C., and Wierzbicki, T.: Malaysia Airlines Flight MH370: Water entry of an Airliner, Notices AMS, 62, 330-344, 2015.

de la Cámara, A., Mancho, A. M., Ide, K., Serrano, E., and Mechoso, C.: Routes of transport across the Antarctic polar vortex in the southern spring, J. Atmos. Sci., 69, 753-767, 2012.

de la Cámara, A., Mechoso, R., Mancho, A. M., Serrano, E., and Ide., K.: Quasi-horizontal transport within the Antarctic polar night vortex: Rossby wave breaking evidence and Lagrangian structures, J. Atmos. Sci., 70, 2982-3001, 2013.

d'Ovidio, F., Fernández, V., Hernández-García, E., and López, C.: Mixing structures in the Mediterranean sea from finitesize Lyapunov exponents, Geophys. Res. Lett., 31, L12203, doi:10.1029/2004GL020328, 2004.

Dritschel, D. G.: Contour dynamics and contour surgery: numerical algorithms for extended, high-resolution modelling of vortex dynamics in two-dimensional, inviscid, incompressible flows, Comput. Phys. Rep., 10, 77-146, 1989.

Farazmand, M. and Haller, G.: Computing Lagrangian Coherent Structures from variational LCS theory, Chaos, 22, 013128, doi:10.1063/1.3690153, 2012.

Froyland, G. and Padberg-Gehle, K.: Almost-invariant and finitetime coherent sets: directionality, duration, and diffusion, Ergodic Theory, Open Dynamics, and Coherent Structures, Proc. Math. Stat., 70, 171-216, 2014.

Froyland, G., Horenkamp, C., Rossi, V., Santitissadeekorn, N., and Gupta, A. S.: Three-dimensional characterization and tracking of an Agulhas Ring, Ocean Model., 52-53, 69-75, doi:10.1016/j.ocemod.2012.05.001, 2012.

Griffa, A., Haza, A., Özgökmen, T. M., Molcard, A., Taillandier, V., Schroeder, K., Chang, Y., and Poulain, P. M.: Investigating transport pathways in the ocean, Deep-Sea Res. Pt. II, 85, 81-95, 2013. 
Grodsky, S. A., Lumpkin, R., and Carton, J. A.: Spurious trends in global surface drifter currents, Geophys. Res. Lett., 38, L10606, doi:10.1029/2011GL047393, 2011.

Haller, G. and Beron-Vera, F. J.: Geodesic theory of transport barriers in two-dimensional flows, Physica D, 241, 1680-1702, 2012.

Haza, A. C., Griffa, A., Martin, P., Molcard, A., Özgökmen, T. M., Poje, A., Barbanti, R., Book, J., Poulain, P., Rixen, M., and Zanasca, P.: Model-based directed drifter launches in the Adriatic Sea: Results from the DART experiment, Geophys. Res. Lett., 34, L10605, doi:10.1029/2007GL029634, 2007.

Hernández-Carrasco, I., López, C., Hernández-García, E., and Turiel, A.: How reliable are finite-size Lyapunov exponents for the assessment of ocean dynamics?, Ocean Model., 36, 208-218, 2011.

Ide, K., Small, D., and Wiggins, S.: Distinguished hyperbolic trajectories in time-dependent fluid flows: analytical and computational approach for velocity fields defined as data sets, Nonlin. Processes Geophys., 9, 237-263, doi:10.5194/npg-9-237-2002, 2002.

Ju, N., Small, D., and Wiggins, S.: Existence and computation of hyperbolic trajectories of aperiodically time-dependent vector fields and their approximations, Int. J. Bif. Chaos, 13, 14491457, 2003.

Kuznetsov, L., Toner, M., Kirwan Jr., A. D., Jones, C. K. R. T., Kantha, L. H., and Choi, J.: The Loop Current and adjacent rings delineated by Lagrangian analysis of the near-surface flow, J. Mar. Res., 60, 405-429, doi:10.1357/002224002762231151, 2002.

Lopesino, C., Balibrea, F., Wiggins, S., and Mancho, A. M.: Lagrangian Descriptors for Two Dimensional, Area Preserving Autonomous and Nonautonomous Maps, Commun. Nonlin. Sci. Numer. Simul., 27, 40-51, 2015.

Madrid, J. A. J. and Mancho, A. M.: Distinguished trajectories in time dependent vector fields, Chaos, 19, 013111, doi:10.1063/1.3056050, 2009.

Mancho, A. M., Small, D., Wiggins, S., and Ide, K.: Computation of Stable and Unstable Manifolds of Hyperbolic Trajectories in Two-Dimensional, Aperiodically Time-Dependent Vectors Fields, Physica D, 182, 188-222, 2003.

Mancho, A. M., Small, D., and Wiggins, S.: Computation of hyperbolic trajectories and their stable and unstable manifolds for oceanographic flows represented as data sets, Nonlin. Processes Geophys., 11, 17-33, doi:10.5194/npg-11-17-2004, 2004.

Mancho, A. M., Hernández-García, E., Small, D., Wiggins, S., and Fernández, V.: Lagrangian transport through an ocean front in the North-Western Mediterranean Sea, J. Phys. Oceanogr., 38, 1222-1237, 2006a.

Mancho, A. M., Small, D., and Wiggins, S.: A comparison of methods for interpolating chaotic flows from discrete velocity data, Comput. Fluids, 35, 416-428, 2006b.

Mancho, A. M., Small, D., and Wiggins, S.: A tutorial on dynamical systems concepts applied to Lagrangian transport in oceanic flows defined as finite time data sets: Theoretical and computational issues, Phys. Rep., 237, 55-124, 2006c.

Mancho, A. M., Wiggins, S., Curbelo, J., and Mendoza, C.: Lagrangian descriptors: A Method for Revealing Phase Space Structures of General Time Dependent Dynamical Systems, Commun. Nonlin. Sci. Numer. Simul., 18, 3530-3557, 2013.
Maxey, M. R. and Riley, J. J.: Equation of motion for a small rigid sphere in a nonuniform flow, Phys. Fluids, 26, 883-889, doi:10.1063/1.864230, 1983.

Mendoza, C. and Mancho, A. M.: The hidden geometry of ocean flows, Phys. Rev. Lett., 105, 038501, doi:10.1103/PhysRevLett.105.038501, 2010.

Mendoza, C. and Mancho, A. M.: Review Article: "The Lagrangian description of aperiodic flows: a case study of the Kuroshio Current”, Nonlin. Processes Geophys., 19, 449-472, doi:10.5194/npg-19-449-2012, 2012.

Mendoza, C., Mancho, A. M., and Rio, M.-H.: The turnstile mechanism across the Kuroshio current: analysis of dynamics in altimeter velocity fields, Nonlin. Processes Geophys., 17, 103-111, doi:10.5194/npg-17-103-2010, 2010.

Mendoza, C., Mancho, A. M., and Wiggins, S.: Lagrangian descriptors and the assessment of the predictive capacity of oceanic data sets, Nonlin. Processes Geophys., 21, 677-689, doi:10.5194/npg-21-677-2014, 2014.

Mezic, I. and Wiggins, S.: A method for visualization of invariant sets of dynamical systems based on the ergodic partition, Chaos, 9, 213-218, 1999.

Mezic, I., Loire, S., Fonoberov, V. A., and Hogan, P. A.: A New Mixing Diagnostic and Gulf Oil Spill Movement, Science, 330, 486-489, 2010.

Nese, J. M.: Quantifying local predictability in phase space, Physica D, 35, 237-250, 1989.

Olascoaga, M. J., Beron-Vera, F., Haller, G., Trinanes, J., Iskandarani, M., Coelho, E. F., Haus, B. K., Huntley, H. S., Jacobs, G., Kirwan Jr., A. D., Lipphardt Jr., B. L., Ozgokmen, T. M., Reniers, A., and Valle-Levinson, A.: Drifter motion in the Gulf of Mexico constrained by altimetric Lagrangian Coherent Structures, Geophys. Res. Lett., 40, 6171-6175, 2013.

Rempel, E. L., Chian, A. C.-L., Brandenburg, A., Munuz, P. R., and Shadden, S. C.: Coherent structures and the saturation of a nonlinear dynamo, J. Fluid Mech., 729, 309-329, 2013.

Rypina, I. I., Scott, S. E., Pratt, L. J., and Brown, M. G.: Investigating the connection between complexity of isolated trajectories and Lagrangian coherent structures, Nonlin. Processes Geophys., 18, 977-987, doi:10.5194/npg-18-977-2011, 2011.

Saab, C.: What's our role in the search for missing flight MH370?, available at: http://csironewsblog.com/2014/03/28/ whats-our-role-in-the-search-for-missing-flight-mh370/ (last access: 29 July 2015), 2014.

Shadden, S. C., Lekien, F., and Marsden, J. E.: Definition and properties of Lagrangian Coherent Structures from finite-time Lyapunov exponents in two-dimensional aperiodic flows, Physica D, 212, 271-304, 2005.

Shadden, S. C., Lekien, F., Paduan, J. D., Chavez, F. P., and Marsden, J. E.: The correlation between surface drifters and coherent structures based on high-frequency radar data in Monterey Bay, Deep-Sea Res. Pt. II, 56, 161-172, 2009.

Sulman, M. H. M., Huntley, H. S., Lipphardt Jr., B. L., Jacobs, G., Hogan, P., and Kirwan Jr., A. D.: Hyperbolicity in temperature and flow fields during the formation of a Loop Current ring, Nonlin. Processes Geophys., 20, 883-892, doi:10.5194/npg-20-8832013, 2013.

Tallapragada, P. and Ross, S. D.: Particle segregation by Stokes number for small neutrally buoyant spheres in a fluid, Physical Rev. E, 78, 036308, doi:10.1103/PhysRevE.78.036308, 2008. 
Tew Kai, E., Rossi, V., Sudre, J., Weimerskirch, H., López, C., Hernández-García, E., Marsac, F., and Garçon, V.: Top marine predators track Lagrangian Coherent Structures, P. Natl. Acad. Sci. USA, 106, 8245-8250, 2009.
Wiggins, S. and Mancho, A. M.: Barriers to transport in aperiodically time-dependent two-dimensional velocity fields: Nekhoroshev's theorem and "Nearly Invariant" tori, Nonlin. Processes Geophys., 21, 165-185, doi:10.5194/npg-21-165-2014, 2014. 